\title{
Malware Detection Using Remedimorbus Application
}

\author{
Prof. C. Ranjeeth Kumar \\ Assistant Professor (Sr.Gr), Department of Information Technology, \\ Sri Ramakrishna Engineering College, Coimbatore
}

\begin{tabular}{|c|c|}
\hline Article History & Abstract \\
\hline $\begin{array}{l}\text { Article Submission } \\
30 \text { November } 2019 \\
\text { Revised Submission } \\
28 \text { Feb } 2020 \\
\text { Article Accepted } \\
17 \text { March } 2020 \\
\text { Article Published } \\
31^{\text {st }} \text { March } 2020\end{array}$ & $\begin{array}{l}\text { As a huge number of new malware tests rise each day, traditional malware } \\
\text { recognition strategies are not sufficient. Static examination strategies, for example, } \\
\text { report signature, fail to recognize obscure projects. Dynamic examination techniques } \\
\text { have low execution and over the top bogus positive charge. A discovery method that } \\
\text { could adjust to the quickly changing malware condition is required. The paper } \\
\text { introduced a spic and span malware identification approach the utilization of } \\
\text { machine picking up information on. This paper proposes an answer where some of } \\
\text { the gadget contemplating calculations are chosen. Utilizing the chose abilities, an } \\
\text { incorporated methodology has been progressed with the picked calculations so the } \\
\text { grouping and identification expense may improve contrasted with static and dynamic } \\
\text { approach. The analyzed malwares equipped with various algorithms and capacities } \\
\text { is utilized for higher order and discovery result. The final product got during utilizing } \\
\text { calculations like Random woodland, Decision tree and Adaboost demonstrates a } \\
\text { precision of } 99.35 \% \text { the use of the Random backwoods, } 98.96 \% \text { the use of Decision } \\
\text { tree and } 98.54 \% \text { utilizing Adaboost. Looking at the static and dynamic strategy, this } \\
\text { incorporated technique gives higher exactness. } \\
\text { Keywords: Malware, gadget,decision tree, adaboost }\end{array}$ \\
\hline
\end{tabular}

\section{Introduction}

Malware is any product intentionally intended to make hurt a PC, server, customer, or PC organize. A wide kind of styles of malware exist, including PC infections, worms, Trojan ponies, ransomware, spyware, adware, rebel programming program, and scareware. They can unfurl wildly, negative a machine's center usefulness and erasing or undermining records. They typically appear as an executable record. Machine picking up information on (ML) is the investigation of PC calculations that upgrade naturally through understanding. It is obvious as a subset of man-made reasoning. Machine contemplating calculations assemble a scientific model dependent on test measurements, perceived as "preparing realities", which will settle on expectations or decisions with out being unequivocally modified to do as such. AI calculations are used in a wide sort of projects, which incorporate email separating and pc vision, where it is extreme or infeasible to create customary calculations to play out the needed obligations.

Machine learning is firmly connected with computational insights, which makes a claim to fame of utilizing PCs. The look at of scientific enhancement guarantees techniques, standard and utility spaces to the field of framework picking up information on. Information mining is a related territory of examine, that spend significant time in exploratory realities investigation through unaided learning. In its product across business issues, gadget contemplating is additionally called prescient examination.

Malware is the most brief term utilized for malevolent programming which is a perilous vindictive bit of code. Malware's motivation is to hurt PC or steal realities from gadget by utilizing abusing vulnerabilities in current assurance framework. Malwares are quickly developing with the progression of time and we can sort malware in to exceptional classes with regards to their practices.The malware might be a content, executable parallel or another bit of code, which have pernicious goal. The first aspirations of malware are to pick up get admission to of gadget, upset device administrations, disavowal of administration, scouse acquire classified insights and 
decimation of assets. At some point malware is certainly not a faulty programming however some authentic programming system will have malware inside it.

Real programming program regularly goes about as covering for malware. Downloading substantial programming program from any web webpage may likewise down burden noxious programming itself. For the most part malwares are situated in broke programming and pilfered programming program. Malwares are not best executable codes however now and then they go about as downloader for malware e.g. PDF and PHP hyperlink which increases control of contraption and down burden extra pernicious programming to execute on gadget. Some product program's favorable position over see of gadget and do a little authentic work so we can not group them malevolent. There are such malwares, which can be marked into Virus, Trojan pony, Adware, Worm and Backdoor. Some of malwares can not be grouped into one classification, on the grounds that malwares have different qualities which compose them in numerous classes and at some point, we known as them sum up malware.

Malwares are examined on establishment of static notwithstanding powerful highlights. In excess of 2300 capacities are removed from dynamic assessment and 92 capacities are extricated statically from twofold report the utilization of PEFILE. Distinctive powerful abilities mixes are utilized for assessment. Four types of dynamic abilities are utilized for malware investigations that are Registry, DLLs, APIs and rundown realities. Machine becoming acquainted with is done on those dynamic component's blends. To see the malware over the network requires broad human mediation, which now and again can likewise cause absence of foreseeing the malware reports. To spare you this, a mechanized programming must be created which distinguishes the malware records without human intercession. This computerized programming need to work dependent on the contraption concentrating so it very well may be finished without human intercessions.

In this manner, malware security of PC frameworks is one of the most critical digital well being obligations regarding unmarried clients and organizations, given that even a solitary assault can realize traded off data and adequate misfortunes. A portion of the applications are it can be utilized in private PCs, riding focus, Organizations to keep up their database ensured, banking Sectors to maintain a strategic distance from attack by means of vindictive documents and in IT enterprises to spare you loss of information through system ambush.

\section{Existing techniques}

In this stage, we will examine the current works and the thought in the rear of static and dynamic assessment for malware class. Z.Salehi et al. [1] proposed distinguishing and dissecting malwares dependent on API calls. The inspect recommended that malwares having comparable direct will name the indistinguishable arrangement of API'S and the equivalent arrangement of contentions. The strategy was to separate trademark vectors the utilization of dynamic assessment method. To decrease the wide assortment of abilities, different capacity decision calculations are utilized. Creators utilized the WINAPIoverride32 gadget in a VMware-fundamentally based virtual framework for the block attempt of API calls. For arrangement, the creators utilized Weka Classifiers [2]. Zane Markel et al. [3] proposed the idea of examination the malwares utilizing the metadata, in the fundamental the headers fragment, and the import report area of the home windows Portable Executable (PE) document group [4]. The creators exceptionally focused on the possibility that the metadata of the noxious executable records varies from the simple executables. Different capacities of PE32 headers are investigated and best those capacities are chosen which are generally fitting for arrangement.

Schultz et al. [5] brought the identification of malwares the utilization of gadget contemplating. The creators extricated specifically three static capacities the utilization of the static investigation strategy which are Portable executable (PE), byte-assortment $\mathrm{n}$-grams approach, and string realities. Inside the 32-piece executable, there are dynamic connection libraries (dll's) from where the highlights are separated. To accomplish better discovery charge, the n-gram approach is utilized wherein the arrangement of n-bytes of string measurements are separated. The string realities gives all the literary substance strings that are encoded in the executables. The 
creators found that their discovery costs are a lot higher the utilization of gadget learning as in contrast with conventional mark based strategies.

Tian et al. [6] utilized the idea of capacity extraction based on various bytes present in the source code of malevolent executable. Utilizing this strategy, they got different capacities from malevolent executables and the recurrence in their occurrence using dynamic investigation and afterward use it to remove various ascribes to hit upon the malignant archive. For muddled records, the executable's codes had been covered up. For class, device becoming more acquainted with calculations found in WEKA are applied. Kotler et al. [7] likewise utilized the possibility of $\mathrm{n}$-grams method gave higher class results. The creators managed various classification calculations and choice tree-based calculation gave better recognition charge. Ahmed et al. [8] separated highlights the utilization of dynamic assessment consolidating each spatial and fleeting records to be had in run time home windows Application Programming Interface (API). As per the creators, dissecting both spatial and worldly capacities in equal can upgrade the discovery charge of the malwares.

Islam et al. [9] chipped away at the joining of static and dynamic assessment of malwares. Capacity period recurrence and printable string data (PSI) vectors are removed the utilization of static examination. Dynamic component vectors are separated from the $\log$ records of dynamic investigation apparatuses along with HOOKAPI and so forth which incorporate API capacities and its boundaries. By and large, the author accumulated the component vectors gained from dynamic and static investigation and mixed them to shape the element vector for the incorporated technique. The outcomes show the precision utilizing incorporated method is higher in contrast with static and dynamic procedure. Dhammi et al. [10] separated powerful capacities from the CSV record produced from the cuckoo sandbox. The creators additionally offered significance to library changes, arrange assessment, muteness, and report subtleties rather concentrating just on API calls. In spite of the fact that each creator endeavored to upgrade the recognition rate and class of the malware yet at the same time there is need for additional improvement in the examination technique. In the later section, we will examine the imperatives of explored writing and our inspiration to adjust new technique for malware investigation.

An infection mark is the unique mark of a pestilence. It is a rigid of extraordinary realities, or bits of code, that permit it to be recognized style of infections can likewise have the equivalent infection signature permitting hostile to infection bundles to unearth various infections when looking out a solitary infection signature. Due to this sharing of a similar infection signature between various infections, hostile to infection projects can once in a while distinguish a destructive ailment that isn't in every case even respected at this point. Ordinarily, new infections have an infection signature that isn't utilized by different infections, anyway new "strains" of perceived infection every so often utilize a similar infection signature as prior strains. The infections are recognized through various techniques, for example, filtering, respectability checking, block attempt, and heuristic discovery. Of these, checking and capture are not surprising, with the elective two just not abnormal in less generally utilized enemy of infection bundles. Some product can likewise wind up obsolete naturally. There isn't any web based filtering accessible inside the product. As a result of this refreshing infection data base should not be possible.

\section{Proposed Methodology}

The location of antivirus done through AI is that the customary strategies neglect to show precision and right discovery of infections may unrealistic through the single recognition of infection records. The proposed method of distinguishing infections empowers us to identify the infections with most precision through single recognition and the machine prepares the everyday troubles and shields the framework from progressing viral assaults from different potential ways as shown in Figure1. The proposed AI based identification utilizes straight relapse, Random woodland, Decision tree, AdaBoost, Gaussian procedure and slope boosting. A product named Remedi Morbusis created, which is a web application utilizes AI models to break down the record is pernicious or not. It recognizes just the.exe record and says precisely whether it is vindictive or not. It distinguishes the new malware as it adapts consistently utilizing AI calculations. 


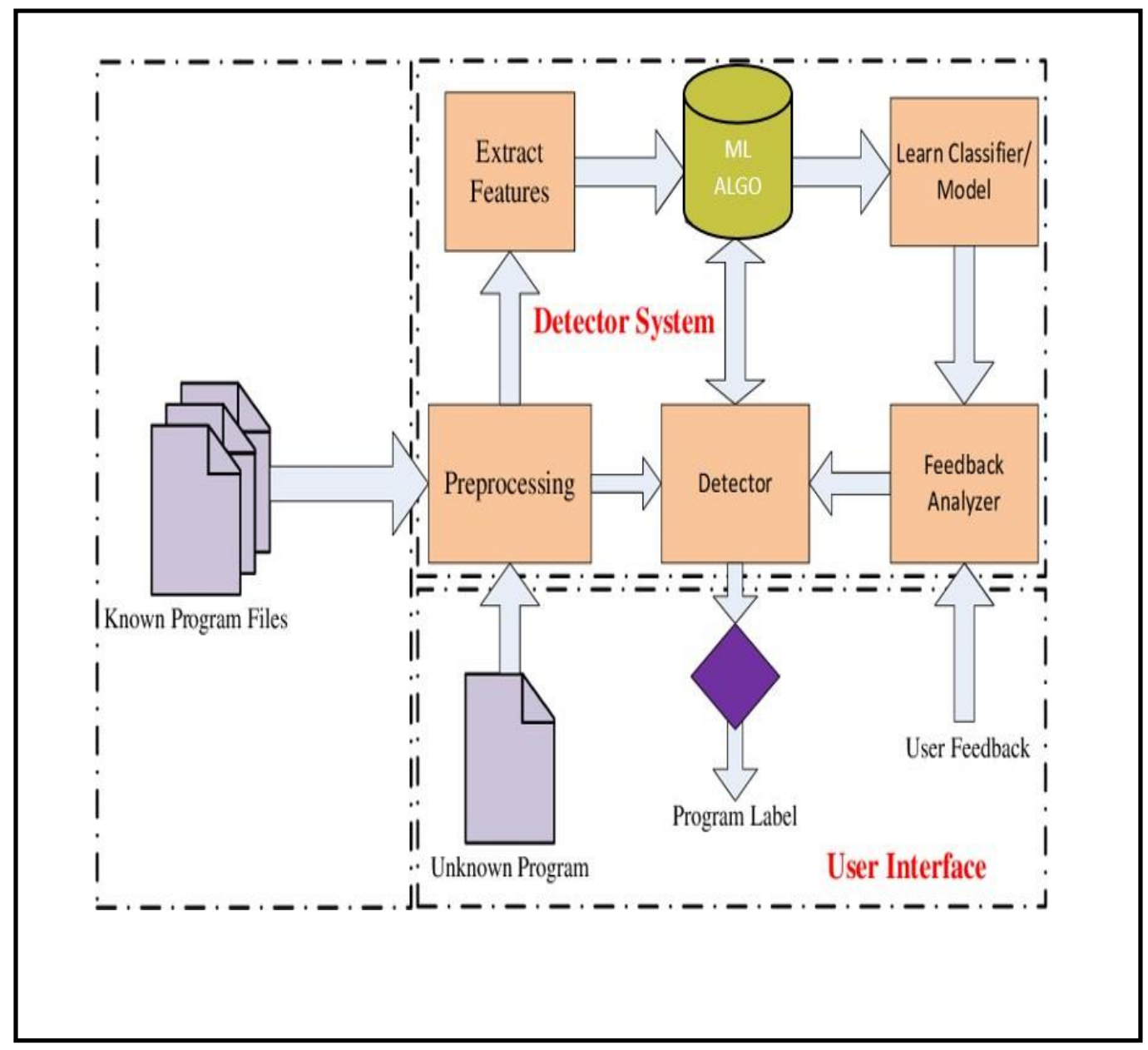

Fig 1: Proposed Malware Detection System

A structure for malware discovery planning to get as scarcely any bogus positives as could be expected under the circumstances, by utilizing a basic and a basic multi-stage mix (course) of various renditions calculations Other computerize order calculations could likewise be utilized in this system, yet we don't investigate here this other option as shown in Figure 2. The Remedi Morbus programming is given as a website page. In the site page the client can transfer or drag the documents in the page for malware recognition. When the document is transferred and the client presses the beginning recognition button, the page sidetracks to another page showing the sort of the record (pernicious or genuine) transferred in the website page

The principle steps performed through this framework are portrayed as follows:

1. A lot of highlights is registered for each twofold record in the preparation or test datasets, in light of numerous potential methods of dissecting a malware.

2. A machine learning system based firstly on one-side dperceptron's and then on include planned uneven perceptron's and a kernelized uneven perceptron's, joined with highlight choice dependent on the F1 and F2 scores, is prepared on a medium-size dataset comprising of clean and malware records. Cross-approval is then acted so as to pick the correct qualities for boundaries. At long last, tests are performed on another, non-related dataset. The got outcomes were extremely reassuring.

3. At long last we will investigate various angles engaged with the scale-up of our system to distinguishing malware documents on huge preparing informational indexes. 


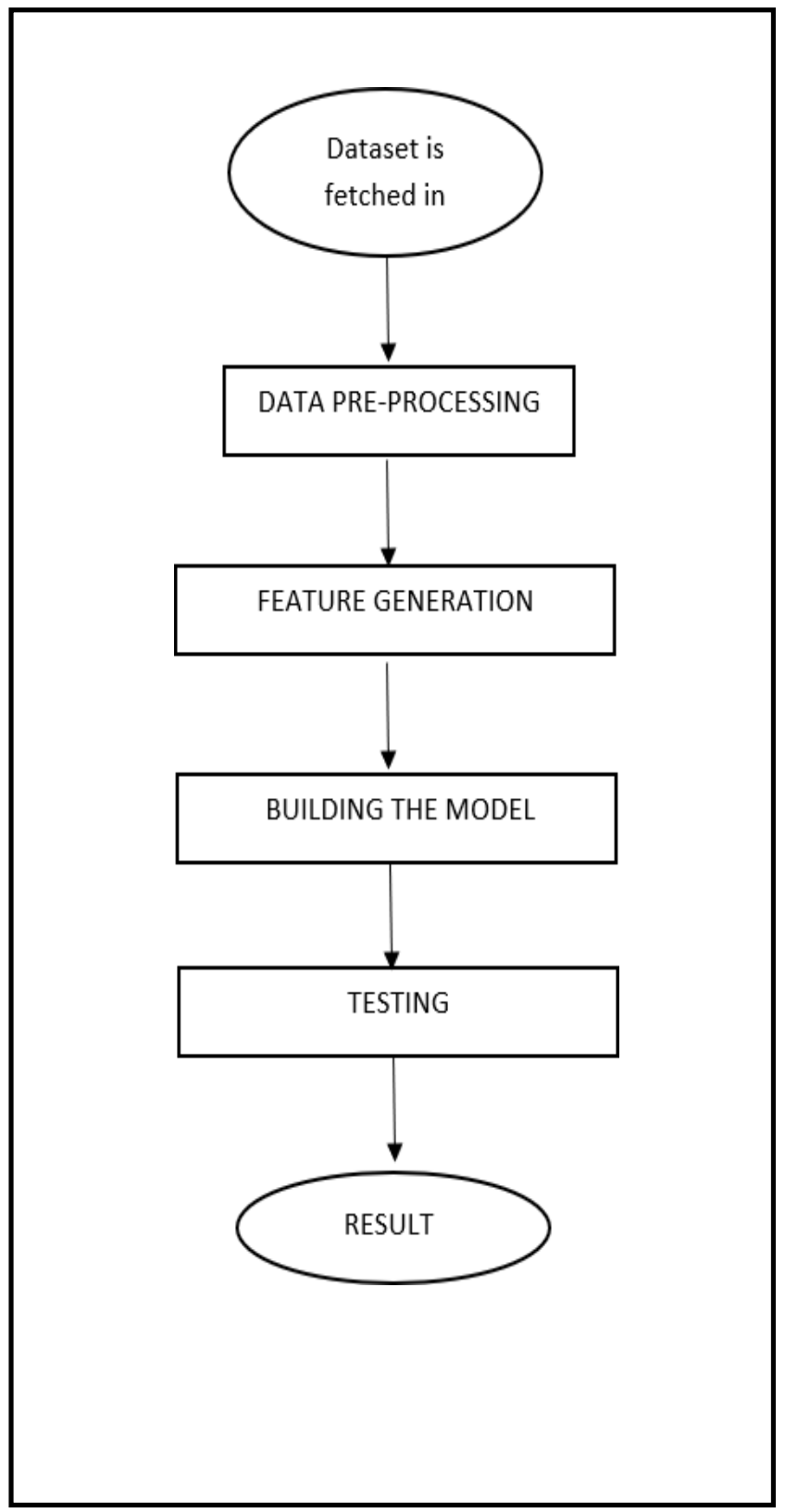

Fig 2: Flow Diagram of Proposed Malware Detection System

Information pre handling is a procedure of setting up the crude data and making it proper for a machine acing model. It is the essential and basic advance while making a machine examining model. Information pre preparing is required obligations for cleaning the data and making it fitting for a gadget learning form which furthermore will build the exactness and effectiveness of a gadget examining adaptation. Certifiable realities as often as possible has missing qualities. In that manner realities. Csv moreover make them miss esteems. Information may have missing qualities for some of thought processes along with perceptions that were not, at this point recorded and measurements defilement.

Taking care of lacking data is fundamental the same number of framework considering calculations do no longer help measurements with missing qualities. : $\mathrm{NaN}$ (an abbreviation for Not a Number), is an interesting gliding factor esteem analyzed by methods for all structures that utilization the typical IEEE skimming point portrayal. Notice that NumPy picked a neighborhood coasting point type for this cluster: which implies dislike the item exhibit from previously, this exhibit underpins rapid activities drove into aggregated code. You must be 
cognizant that $\mathrm{NaN}$ is a piece like a realities infection it taints some other thing it contacts. Despite the activity, the consequence of arithmetic with $\mathrm{NaN}$ can be another NaN. Having missing qualities in an informational collection can cause mistakes with a couple of framework examining calculations. The best methodology for overseeing missing insights is to remove records that contain a missing worth or by crediting the information. Attributing alludes to utilizing a model to supplant lacking qualities.

Machine learning algorithms are the engines of machine learning, meaning it is the algorithms that turn a data set into a model. The malware detection involving machine learning involves different algorithms to obtain the best result and accuracy from these algorithms used. The following are the different algorithms which are used
a) Linear regression
b) Random forest
c) Decision tree
d) Ada Boost
e) Gaussian process
f) Gradient boosting

Straight relapse is the quantization the association of two factors. One variable is utilized to acquire the yield this is each other variable. The yield is reliant on the two discrete coefficients especially the intercept (a)and the slope(b). The captures are the component inside the chart speaks to the coordinating of dataset with expected components to gain the malware record. The info variable $\mathrm{x}$ is relegated for elements of the dataset. The enter variable $\mathrm{y}$ is doled out as substantial worth. The expectation of genuine (y) is from the highlights (x).The capture an is the point wherein $\mathrm{x}$ and $\mathrm{y}$ get together. The realities guides nearer toward the relapse line ( $\mathrm{b}$ incline) are the focuses with the top notch exactness. Though the data factors from the line of relapse are mistaken normally named exceptions as they don't fit near to the relapse line and needs precision.

Testing is an examination led to presents take holders with realities around the high-caliber of the product program administration or item under investigate. Programming testing can likewise offer a target, unprejudiced perspective on the product program to permit the business to acknowledge and capture the perils of programming execution. Test procedures incorporate the way toward executing a program or utility with the aim of discoveries of tware bugs (errorsorotherdefects), and confirming that the product item is fit as a fiddle for use.

Cross approval is executed to separate the dataset into arbitrary train and check subsets. Test_size $=0.2$ speak to the level of the data set to incorporate inside the test split. Train_size $=0.8$ speak to the portion of the dataset to include inside the instruct split. Train_Split_Data of scikit-learn procedure is the method used to physically the train and investigate realities. Cross_val_score (returns rating of each investigate folds)/Cross_predict_score (restores the anticipated score for every announcement in the input dataset when it was a piece of the test set) from the scikit_learn library.

\section{Simulation Results}

From survey on subject of Malware Analysis with Machine Learning, it is inferred that this antivirus can be executed with different AI calculations and gives the usefulness as checking if the application is pernicious or not a shown in Figure 3. By utilization of this framework will decrease loss of information. By inspecting various papers on AI calculations, it very well may be inferred that malware examination can be simpler by utilizing the successful calculation and can bring about characterization of the record into genuine or vindictive dependent on the best exactness among the applied calculation as shown in Table1.In light of the exactness of the above calculations, the best precision calculation is picked and the outcome is shown. 
Table 1 : Accuracy rates of algorithms

\begin{tabular}{|c|c|}
\hline ALGORITHMS & ACCURACY \\
\hline DECISION TREE & $98.96 \%$ \\
\hline RANDOM FOREST & $99.35 \%$ \\
\hline ADABOOST & $98.54 \%$ \\
\hline GRADIENT BOOSTING & $98.73 \%$ \\
\hline GNB & $70.24 \%$ \\
\hline LINEAR REGRESSION & $59.01 \%$ \\
\hline
\end{tabular}

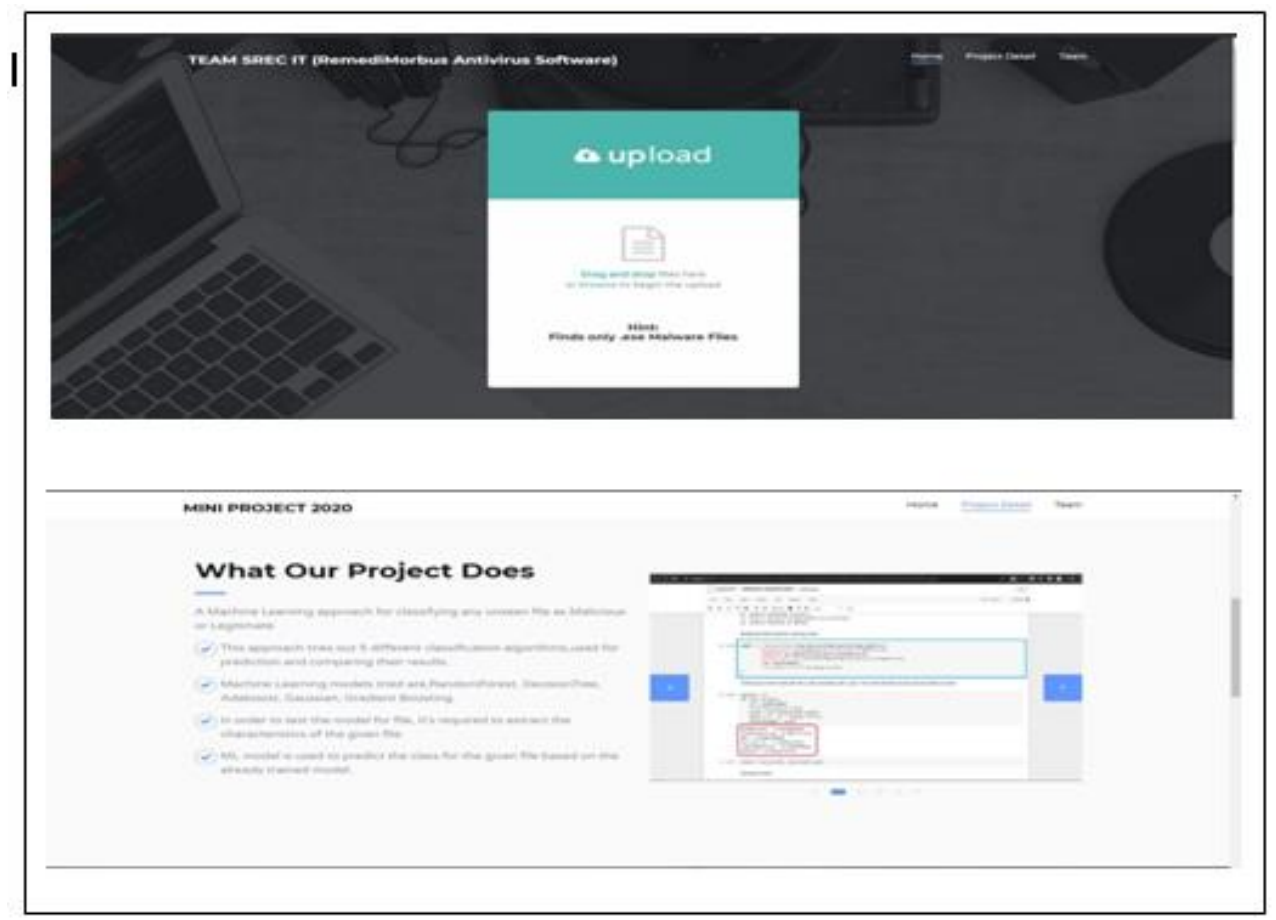

\section{Conclusion}

Fig 5: Remedi morbus software

The progression in innovation is a nonstop procedure. This application is utilized by different clients to safeguard the machine secured and safe. This malware examination is quicker as it filters just a solitary .Exe augmentation record at once with the goal that it will sparing the investing energy in checking them. In this endeavor different contraption picking up information on calculations are utilized wherein as an absolute last it outcomes the kind of document is real or not legitimate. By utilization of this malware examination the data in 
the client machine might be secured. Any buyer can get section to this web website page as it is an open flexibly and the same membership is required. We have achieved better location cost for incorporated method when contrasted with static and dynamic assessment approach for the entirety of the sort calculations we have utilized. The outcomes likewise show that Random woodland grouping calculation is higher for class of themalware informational index we have gathered in light of the fact that it gives higher precision when contrasted with various classifiers. In light of the examination, tips proposed for the further look at are as per the following: Develop a various leveled multi-class acing way to deal with beautify the testing productivity when the quantity of malware classes will turn out to be incredibly gigantic. Identification exactness can be improved, through correspondingly concentrates into arrangement calculations and approaches to stamp malware measurements more prominent precisely. For the not so distant future we intend to coordinate more class calculations to it, for example huge edge perceptron's and Support Vector Machines

\section{References}

[1] Z. Salehi, M. Ghiasi, and A. Sami. A miner for malware detection based on API function calls and their arguments. 16th CSI International Symposium on Artificial Intelligence and Signal Processing (AISP), pp. 563-568, May2012.

[2] WEKA version 3.8.1, the University of Waikato, Available at: http://www.cs.waikato.ac.nz/ml/weka/(Accessed: February,2017).

[3] Z. Markel and M. Bilzor, "Building a machine learning classifier for malware detection," 2014 Second Workshop on Anti-malware Testing Research(WATeR),Canterbury,pp.110.1109/WATeR.2014.7015757, 2014.

[4] E. Carrera. Pefile python module, 2014[online]. Accessed September 2016.Available:https://pypi.python.org/pypi/pefile/

[5] Schultz MG, Eskin E, Zadok E, Stolfo SJ. Data mining methods for detection of new malicious executables. In: Proceedings of the 2001IEEE symposium on security and privacy. Washington, DC, USA; IEEE Computer Society; pp. 38-49,2014.

[6] Tian, R., Batten, L. and Versteeg, S. (2008) "Function Length as a Tool for Malware Classification". Proceedings of the 3rd International Conference on Malicious and Unwanted Software, Fairfax, pp.5764, 7-8 October2008,

[7] Kolter, J. and Maloof, M. "Learning to Detect Malicious Executables in the Wild". Proceedings of the 10th ACMSIGKDD International ConferenceonKnowledgeDiscoveryandDataMining,pp470-478,2004.

[8] Ahmed F, Hameed H, Shafiq MZ, Farooq M. Using spatio-temporal information in API calls with machine learning algorithms for malware detection. In: AISec'09: proceedings of the 2nd ACM workshop on Securityandartificialintelligence.NewYork,NY,USA:ACM;P.55-62, 2009.

[9] R.Islam,R.Tian,L.M.Batten,andS.Versteeg.Classificationofmalware based on integrated static and dynamic features. Journal of Network and Computer Applications. vol. 36, pp. 646-656,2013.

[10] A.Dhammiand M.Singh,"Behavior analysis of malware using machine learning,"2015 Eighth International Conference on Contemporary Computing (IC3), Noida, 2015, pp. 481486.doi:10.1109/IC3.2015.7346730 